\title{
Benzene substituted with bipyridine and terpyridine as electron-transporting materials for organic light-emitting
}

\section{devices}

Musubu Ichikawa ${ }^{1,2, *}$, Takayuki Yamamoto ${ }^{1}$, Hyeon-Gu Jeon ${ }^{1}$, Kouki Kase ${ }^{3}$, Shuichi Hayashi $^{3}$, Makoto Nagaoka ${ }^{3}$, Norimasa Yokoyama ${ }^{3}$

${ }^{1}$ Functional Polymer Science Course, Division of Chemistry and Materials, Faculty of Textile Science and Technology, Shinshu University, 3-15-1 Tokida, Ueda, 386-8567, Japan

${ }^{2}$ Presto, Japan Science and Technology Agency (JST), 4-8-1 Honcho, Kawaguchi, Saitama, 332-0012, Japan

${ }^{3}$ Hodogaya Chemical Co., Ltd., 45 Miyukigaoka, Tsukuba, Ibaraki, 305-0841, Japan

New electron-transporting materials for organic light-emitting devices (OLEDs) based on trisubstituted benzene with both bipyridine and terpyridine, 1,3-bisbipyridyl-5terpyridylbenzene (BBTB) and 1-bipyridyl-3,5-bisterpyridylbenzene (BTBB), were developed. Glass transition temperatures of BBTB and BTBB were $93{ }^{\circ} \mathrm{C}$ and $108{ }^{\circ} \mathrm{C}$, respectively, and BTBB was completely amorphous with no melting point. Electron mobilities of BTBB exceeded the order of $10^{-4} \mathrm{~cm}^{2} / \mathrm{Vs}$, while those of BBTB were very high and reached $10^{-3} \mathrm{~cm}^{2} / \mathrm{Vs}$ at an electric field of approximately $500 \mathrm{kV} / \mathrm{cm}^{2}$. These 
high mobilities contributed to a low voltage operation. For example, in the case of the conventional aluminum trisquinolinol (Alq)-based fluorescent OLED with BTBB, current densities of $3.5 \mathrm{~mA} / \mathrm{cm}^{2}$ and $100 \mathrm{~mA} / \mathrm{cm}^{2}$ were reached at voltages of $3.0 \mathrm{~V}$ and 4.5 $\mathrm{V}$, respectively. In addition, ionization potentials of BBTB $(6.33 \mathrm{eV})$ and BTBB $(6.50 \mathrm{eV})$ were sufficiently large to confine holes in common emissive layers.

Keywords: electron-transporting material, organic light-emitting device, pyridine, electron mobility, hole-blocking, glass transition temperature 


\section{Introduction}

Organic light-emitting devices (OLEDs) are of potential interest in applications such as flat televisions, mobile displays ${ }^{1,2}$, lights ${ }^{3}$, and optical communication light sources. ${ }^{4}$ OLEDs are usually composed of functionally divided organic multilayersfor example, hole-transporting (HT), emissive, and electron-transporting (ET) layers. ${ }^{5,6}$ In the last decade, many kinds of amorphous molecular semiconductor materials, ${ }^{7,8} \mathrm{HT}$ materials, ${ }^{9-16}$ and ET materials (ETMs), ${ }^{17-28}$ have been proposed.

Recently, it has been reported that pyridine-based materials work well as ETMs with high electron mobility for OLEDs. For example, Kido et al. reported that three compounds based on triphenylbenzene with three pyridines were very good ETMs for OLEDs, and in particular, the compound in which pyridine is substituted at the 4 position showed extremely high electron mobility above $10^{-3} \mathrm{~cm}^{2} / \mathrm{Vs} .{ }^{29}$ We also reported that trisubstituted benzenes with bipyridine or terpyridine were good ETMs, and tris(bipyridyl)benzene showed high electron mobility above $10^{-4} \mathrm{~cm}^{2} / \mathrm{Vs} .{ }^{30}$ However, the glass transition temperature $\left(T_{\mathrm{g}}\right)$ of the materials was only $75^{\circ} \mathrm{C}$ and insufficient for practical use. In contrast, tris(terpyridyl)benzene showed a much higher $T_{\mathrm{g}}$ of $110{ }^{\circ} \mathrm{C}$, which is greater than the general criterion of thermal stability for practical use, but the electron mobility of the material decreased to an order of $10^{-5} \mathrm{~cm}^{2} / \mathrm{Vs}$. Large bulkiness of a terpyridyl substituent might lead to a sufficient increase in $T_{\mathrm{g}}$, but it also leads to a sizable decrease in the electron mobility. Here we demonstrate that asymmetrically substituting benzene with bipyridine and terpyridine can be useful for overcoming this trade-off, and we achieve a high mobility and $T_{\mathrm{g}}$ of greater than $10^{-3} \mathrm{~cm}^{2} / \mathrm{Vs}$ and $100{ }^{\circ} \mathrm{C}$, respectively. 


\section{Experimental}

\section{Materials}

Figure 1 shows chemical structures of the newly developed materials, and Scheme 1 shows their synthetic routes; these materials were synthesized step-by-step by the Suzuki-Miyaura coupling reaction with bipyridine and terpyridine bromides.

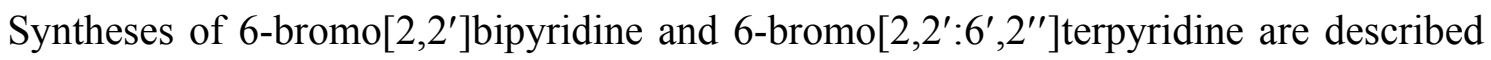
in detail elsewhere. ${ }^{30}$ BBTB and BTBB were purified by column chromatography and temperature-gradient sublimation in a flow stream of pure Ar gas. Chemical structures

were confirmed by ${ }^{1} \mathrm{H} \mathrm{NMR},{ }^{13} \mathrm{C} \mathrm{NMR}$, and mass spectroscopy. Other OLED materials were obtained from industrial companies as sublimation grades and used without further purification.

\section{Synthesis}

Synthesis of BBTB: First, (3,5-Dibromophenyl)boronic acid (2.69 g, $9.61 \mathrm{mmol})$ was treated with bromoterpyridine $(3.0 \mathrm{~g}, 9.61 \mathrm{mmol})$ by the Suzuki-Miyaura coupling reaction using $\mathrm{K}_{2} \mathrm{CO}_{3}(3.98 \mathrm{~g}, 28.83 \mathrm{mmol})$ with pure water $(14.4 \mathrm{~mL})$ as the base, tetrakis(triphenylphosphine)palladium(0) $(0.835 \mathrm{~g}, 0.72 \mathrm{mmol})$ as the catalyst, and a $4: 1$ toluene-ethanol mixture $(150 \mathrm{~mL})$ as the solvent. The reaction mixture was refluxed with stirring for $22 \mathrm{~h}$ and then cooled to room temperature. Pure water was added and the mixture was stirred for $30 \mathrm{~min}$. The mixture was then extracted using toluene, dried with $\mathrm{MgSO}_{4}$, filtered to remove precipitates, and distilled to remove toluene giving a crude powder (5.64 g). Purification by silica-gel (NH silica, 150 g) column chromatography using a 2:1 cyclohexane-toluene mixture as the eluent yielded the first intermediate (1,3-dibromo-5-terpyridylbenzne, $2.45 \mathrm{~g} ; 54.6 \%$ yield; $98.4 \%$ purity by 
high-performance liquid chromatography (HPLC)). Then, the obtained intermediate (7.98 $\mathrm{g}, 17.1 \mathrm{mmol})$ was treated with bis(pinacolato)diboron $\left(\operatorname{Pin}_{2} \mathrm{~B}_{2} ; 10.41 \mathrm{~g}, 41.0\right.$ mmol), potassium acetate $(10.1 \mathrm{~g}, 103 \mathrm{mmol})$, and $[1,1$ ' bis(diphenylphosphino)ferrocene]dichloropalladium(II) $\quad\left(\mathrm{PdCl}_{2}(\mathrm{dppf}) ; \quad 0.83 \mathrm{~g}, \quad 1.03\right.$ mmol) as the catalyst in dioxane $(240 \mathrm{~mL})$ at $80{ }^{\circ} \mathrm{C}$. After $8 \mathrm{~h}$, the reaction mixture was cooled to room temperature, and then poured into a $3000-\mathrm{mL}$ beaker filled with $1500-$ $\mathrm{mL}$ pure water. After stirring for $40 \mathrm{~min}$, a crude black powder $(10.4 \mathrm{~g})$ was obtained. Adsorption purification with diol-bonded silica (48 g) was carried out using a toluene $(200 \mathrm{~mL})$ solution of the obtained crude powder. The solution was filtered to remove the silica gel, distilled to remove toluene, washed with $150 \mathrm{~mL}$ hexane, and dried at 60 ${ }^{\circ} \mathrm{C}$ under vacuum for $1 \mathrm{~h}$ to give the second intermediate (1,3-bis(pinacorato boryl)-5terpyridylbenzene, $7.87 \mathrm{~g} ; 82.0 \%$ yield; $90.4 \%$ purity by HPLC). The purified second intermediate (7.50 g, $13.4 \mathrm{mmol})$ was treated with bromobipyridine (6.96 g, $29.5 \mathrm{mmol})$ using $\mathrm{K}_{2} \mathrm{CO}_{3}(5.56 \mathrm{~g}, 40.2 \mathrm{mmol})$ with pure water $(21 \mathrm{~mL})$ as the base, tetrakis(triphenylphosphine)palladium(0) $(0.77 \mathrm{~g}, 0.67 \mathrm{mmol})$ as the catalyst, and a $4: 1$ toluene-ethanol mixture $(10.4 \mathrm{~g})$ as the solvent. The reaction mixture was refluxed with stirring for $24 \mathrm{~h}$ and then cooled to room temperature. Pure water was added and the mixture was stirred for $30 \mathrm{~min}$. Then, the mixture was extracted using toluene, dried with $\mathrm{MgSO}_{4}$, filtered to remove precipitates, and distilled to remove toluene giving a crude powder (10.2 g). Adsorption purification by silica-gel column chromatography (NH silica; $40 \mathrm{~g}$ ) was carried out using a toluene solution $(150 \mathrm{~mL})$ of the obtained crude powder, filtered for removing the silica, and distilled to remove toluene. The obtained solid was washed in hexane $(200 \mathrm{~mL})$ and then dried at $60{ }^{\circ} \mathrm{C}$ under vacuum for $2 \mathrm{~h}$. Finally, purification by silica-gel (NH silica, $240 \mathrm{~g}$ ) column chromatography 
using a chloroform-hexane mixture as the eluent, the ratio of which was changed from 1:10 to 1:0 step by step, yielded BBTB (7.69 g; 97\% purity by HPLC). The powder was washed with 2-propyl ether $(200 \mathrm{~mL})$, filtered, and dried at $60^{\circ} \mathrm{C}$ under vacuum for $2 \mathrm{~h}$ to give pure BBTB (5.99 g; 70.0\% yield; $98.6 \%$ purity by HPLC). ${ }^{1} \mathrm{H}$ NMR $(\mathrm{CDCl} 3) \delta_{\mathrm{H}}$ (600 MHz, $\left.\mathrm{CDCl}_{3}\right): 9.03(1 \mathrm{H}, \mathrm{d}, \mathrm{J}=1.4 \mathrm{~Hz}), 9.02(2 \mathrm{H}, \mathrm{d}, \mathrm{J}=2.1 \mathrm{~Hz}), 8.79(2 \mathrm{H}, \mathrm{d}, \mathrm{J}=$ $7.6 \mathrm{~Hz}), 8.76(1 \mathrm{H}, \mathrm{d}, \mathrm{J}=7.6 \mathrm{~Hz}), 8.72(3 \mathrm{H}, \mathrm{t}, \mathrm{J}=4.8 \mathrm{~Hz}), 8.67(2 \mathrm{H}, \mathrm{d}, \mathrm{J}=7.6 \mathrm{~Hz}), 8.64$ $(2 \mathrm{H}, \mathrm{dd}, \mathrm{J}=7.6,1.4 \mathrm{~Hz}), 8.49(2 \mathrm{H}, \mathrm{dd}, \mathrm{J}=7.6,1.4 \mathrm{~Hz}), 8.46(1 \mathrm{H}, \mathrm{dd}, \mathrm{J}=7.6,1.4 \mathrm{~Hz})$, 8.02-7.95 (8H, m), 7.88-7.85 (3H, m), 7.36-7.33 (3H, m). $\delta_{\mathrm{C}}\left(150 \mathrm{MHz}, \mathrm{CDCl}_{3}\right)$ : $156.37,156.29,156.21,156.16,155.87,155.81,155.54,155.30,149.15,149.11,140.33$, $140.31,137.82,137.78,137.69,136.85,136.83,126.20,126.18,123.79,123.74,121.35$, 121.25, 121.19, 121.03, 120.61, 120.59, 119.68, 119.61. Mass spectrum: 618.2406 and isotopes $(100 \%) \quad\left([\mathrm{M}+\mathrm{H}]^{+} ; \quad 618.24061894\right.$ for $\left.\mathrm{M}: \mathrm{C}_{41} \mathrm{H}_{28} \mathrm{~N}_{7}\right)$ after sublimation purification.

Synthesis of BTBB: First, (3,5-Dibromophenyl)boronic acid (12.1 g, $43.3 \mathrm{mmol})$ was treated with bromobipyridine $(10.2 \mathrm{~g}, 43.3 \mathrm{mmol})$ using $\mathrm{K}_{2} \mathrm{CO}_{3}(18.0 \mathrm{~g}, 130 \mathrm{mmol})$ with pure water $(130 \mathrm{ml})$ as the base, tetrakis(triphenylphosphine)palladium(0) $(2.50 \mathrm{~g}$, $2.19 \mathrm{mmol})$ as the catalyst, and a $4: 1$ toluene-ethanol mixture $(338 \mathrm{~mL})$ as the solvent. The reaction mixture was refluxed with stirring for $68 \mathrm{~h}$ and then cooled to room temperature. Saturated sodium chloride aqueous solution was added and the mixture was stirred for $30 \mathrm{~min}$. The resulting mixture was extracted using toluene, dried with $\mathrm{MgSO}_{4}$, filtered to remove precipitates, and distilled to remove the toluene giving a grayish-white crude powder. The crude powder was washed in refluxing methanol (600 $\mathrm{mL}$ ) for $1 \mathrm{~h}$. The mixture was cooled to room temperature and filtered. The first 
intermediate (1,3-dibromo-5-bipyridylbenzene, $8.12 \mathrm{~g}$; 48.4\% yield; $76.8 \%$ purity by HPLC) was obtained. The obtained intermediate $(8.12 \mathrm{~g}, 20.9 \mathrm{mmol})$ was treated with $\operatorname{Pin}_{2} \mathrm{~B}_{2}(12.75 \mathrm{~g}, 50.2 \mathrm{mmol})$, potassium acetate $(12.3 \mathrm{~g}, 125.4 \mathrm{mmol})$, and $\mathrm{PdCl}_{2}(\mathrm{dppf})$ $1.02 \mathrm{~g}, 1.25 \mathrm{mmol})$ as the catalyst in dioxane $(240 \mathrm{~mL})$ at $80{ }^{\circ} \mathrm{C}$. After $5.5 \mathrm{~h}$, the reaction mixture was cooled to room temperature and then poured into a $3000-\mathrm{mL}$ beaker filled with $1500-\mathrm{mL}$ pure water. After stirring for $2 \mathrm{~h}$, the mixture was filtered to give a crude powder (11.6 g). Adsorption purification with diol-bonded silica (80 g) was carried out using a toluene solution $(200 \mathrm{~mL})$ of the obtained crude powder. The solution was filtered for removing silica, distilled to remove toluene, and then recrystallized from a 1:1 toluene-hexane mixture to give the second intermediate $(1,3-$ bis(pinacolato boryl)-5-bipyridylbenzene, $5.08 \mathrm{~g} ; 50.3 \%$ yield; $94.5 \%$ purity by HPLC). The purified second intermediate $(4.98 \mathrm{~g}, 10.3 \mathrm{mmol})$ was treated with bromoterpyridine $(7.07 \mathrm{~g}, 22.7 \mathrm{mmol})$ using $\mathrm{K}_{2} \mathrm{CO}_{3}(4.27 \mathrm{~g}, 30.9 \mathrm{mmol})$ with pure water $(16 \mathrm{~mL})$ as the base, tetrakis(triphenylphosphine)palladium $(0)(0.60 \mathrm{~g}, 0.52$ mmol) as the catalyst, and a 4:1 toluene-ethanol mixture $(50 \mathrm{~mL})$ as the solvent. The reaction mixture was refluxed with stirring for $72 \mathrm{~h}$ and then cooled to room temperature. Note that $0.6 \mathrm{~g}$ catalyst was added to the reaction mixture at each elapsed time of 5,21, 27, and $68 \mathrm{~h}$. After filtering off white precipitates, which appear while cooling the mixture in ice, saturated sodium chloride aqueous solution was added to the filtered solution and the mixture was extracted using toluene. The toluene solution was dried with $\mathrm{MgSO}_{4}$ and filtered to remove $\mathrm{MgSO} 4$. The solution was distilled to remove toluene and dried under vacuum to give crude BTBB (14 g). Purification by silica-gel (NH silica, $300 \mathrm{~g}$ ) column chromatography using toluene as the eluent yielded BTBB (6.02 g; 97.5\% purity by HPLC). BBTB was washed with refluxing methanol (100 mL), 
filtered, and dried at $80{ }^{\circ} \mathrm{C}$ under vacuum for $1 \mathrm{~h}$ to give pure BTBB $(2.02 \mathrm{~g} ; 20.0 \%$ yield; $98.4 \%$ purity by $\mathrm{HPLC}) . \delta_{\mathrm{H}}\left(600 \mathrm{MHz}, \mathrm{CDCl}_{3}\right):{ }^{1} \mathrm{H} \mathrm{NMR}(\mathrm{CDCl} 3) \delta: 9.03(2 \mathrm{H}, \mathrm{d}$, $\mathrm{J}=1.4 \mathrm{~Hz}), 9.00(1 \mathrm{H}, \mathrm{t}, \mathrm{J}=1.7 \mathrm{~Hz}), 8.79(1 \mathrm{H}, \mathrm{d}, \mathrm{J}=6.9 \mathrm{~Hz}), 8.76(2 \mathrm{H}, \mathrm{d}, \mathrm{J}=7.6 \mathrm{~Hz})$, 8.74-8.72 (3H, m), 8.68-8.65 (2H, m), $8.49(1 \mathrm{H}, \mathrm{dd}, \mathrm{J}=7.6,1.4 \mathrm{~Hz}), 8.46(2 \mathrm{H}, \mathrm{dd}, \mathrm{J}=$ 7.6, $1.4 \mathrm{~Hz}), 8.03-7.96(7 \mathrm{H}, \mathrm{m}), 7.89-7.86(3 \mathrm{H}, \mathrm{m}), 7.36-7.34(3 \mathrm{H}, \mathrm{m}) . \delta_{\mathrm{C}}(150 \mathrm{MHz}$, $\left.\mathrm{CDCl}_{3}\right): 156.39,156.3,156.19,156.13,155.86,155.80,155.55,155.30,149.15,149.12$, $140.29,140.27,137.83,137.79,137.70,136.86,128.23,126.17,125.23,123.81,123.75$, $121.37,121.26,121.21,121.05,120.60,120.58,119.69,119.63$. Mass spectrum: 695.2672 and isotopes $(100 \%)\left([\mathrm{M}+\mathrm{H}]^{+} ; 695.26716804\right.$ for $\left.\mathrm{M}: \mathrm{C}_{46} \mathrm{H}_{31} \mathrm{~N}_{8}\right)$ after sublimation purification.

\section{Device Fabrication and Measurements}

All OLEDs were fabricated on 150-nm-thick layers of indium-tin oxide (ITO) that were commercially precoated onto glass substrates with a sheet resistance of 14 $\Omega /$ sq. The solvent-cleaned ITO surface was treated with $\mathrm{O}_{2}$ plasma for 5 min and then the substrate was immediately loaded into a high-vacuum chamber (base pressure below $\left.\sim 1 \times 10^{-4} \mathrm{~Pa}\right)$ where organic layers, 0.5 -nm-thick LiF, and 200-nm-thick aluminum cathode layers were deposited by thermal evaporation. Deposition rates were $0.6 \AA / \mathrm{s}$ for organic materials, $0.1 \AA / \mathrm{s}$ for $\mathrm{LiF}$, and $2 \AA / \mathrm{s}$ for $\mathrm{Al}$. The structure of the devices fabricated in this study is shown in Figure 2. The current density-applied voltageluminance $(J-V-L)$ characteristics of the OLEDs were measured by a commercial OLED-characteristics measurement system (Precise Gauge EL1003) and a source meter (Keithley 2400). EL spectra were recorded simultaneously by EL1003 during $J-V-L$ measurements. 
${ }^{1} \mathrm{H}$ and ${ }^{13} \mathrm{C}$ NMR spectra were recorded on a Fourier transform NMR spectrometer (JEOL JNM-ECA600). Mass spectroscopic analysis was carried out on a matrix-assisted laser desorption ionization TOF mass spectrometer (Bruker Daltonics Autoflex) with no internal standard. Thermal analyses were performed on a differential scanning calorimeter (Seiko Instruments DSC-6200) at a heating rate of $10{ }^{\circ} \mathrm{C} / \mathrm{min}$ under $\mathrm{N}_{2}$ gas. UV and visible absorption spectra were recorded on a spectrophotometer (Shimadzu UV-3150); photoluminescence (fluorescence and phosphorescence) spectra were recorded on a spectrofluorometer (Horiba FluoroMax4P). Ionization potential $\left(I_{\mathrm{p}}\right)$ was determined by a photoelectron-emission-yield spectrometer (Riken Keiki AC-3). Optical band gaps were determined by the spectral onset of each UV-visible absorption spectrum; $E_{\mathrm{a}}$ was then estimated by subtracting the band gap energy from $I_{\mathrm{p}}$. Spectroscopic measurements were conducted with thin films prepared by thermal evaporation on quartz substrates. We also determined densities of the thin films prepared on quartz substrates by thermal evaporation using X-ray reflectometry with a Rigaku SmartLab.

Electron mobility was measured by conventional TOF techniques. The excitation light was a 500-ps-duration optical pulse from a $\mathrm{N}_{2}$ gas laser $(\lambda=337 \mathrm{~nm}$, Lasertechnik Berlin MNL 200). Test samples were prepared by thermal evaporation in vacuum and encapsulated with fresh desiccant under a highly inert $\mathrm{N}_{2}$ atmosphere at the $\mathrm{O}_{2}$ concentration of $<20 \mathrm{ppm}$. A 100 -nm-thick fullerene $\mathrm{C}_{60}$ layer was used as the charge-generation layer for optical excitation. ${ }^{31}$ The structure of the TOF samples is shown in Fig. 2.

Computational chemistry was conducted using commercial software (Wavefunction Spartan 10W) on a personal computer with a quad-core processor. 
Density functional theory was used for determining optimized geometries and their energies with a B3LYP hybrid functional. The basis sets $6-31 \mathrm{G}^{*}$ and $6-311+\mathrm{G}^{* *}$ were used to calculate the optimized geometries and their energies, respectively.

\section{Results and Discussion}

\section{Thermal and Electronic Properties}

Figure 3 shows differential scanning calorimetry (DSC) curves of the two ETMs. The materials form amorphous glassy thin films, as glass transition behaviors were observed in the second slow heating after melting followed by quick cooling. That is, we found baseline shifts at $93{ }^{\circ} \mathrm{C}$ for BBTB and $108{ }^{\circ} \mathrm{C}$ for BTBB. BTBB, which has two terpyridines, exhibited a higher glass transition temperature $\left(T_{\mathrm{g}}\right)$ of $108{ }^{\circ} \mathrm{C}$ than BBTB, which has one terpyridine. This is probably because of its larger molecular weight (MW) and the bulkiness of terpyridine. In addition, BTBB is completely amorphous. That is, glass transition occurred and no melting point appeared in the first heating. Furthermore, no recrystallization occurred in the cooling process with a relatively slow descent rate of $-10{ }^{\circ} \mathrm{C} / \mathrm{min}$, and the glass transition behavior could be observed again. All this supports the fact that BTBB is completely amorphous. The $T_{\mathrm{g}} \mathrm{S}$ of these materials increased in comparison with that of tris(bipyridyl)benzene, which, as we reported before, was $75{ }^{\circ} \mathrm{C}$. An obvious reason for the increase in $T_{g}$ is the increase in the molecular weight by adding one or two pyridyl groups. Moreover, the $T_{\mathrm{g}}$ of BTBB was almost the same as that of tris(terpyridyl)benzene $\left(110^{\circ} \mathrm{C}\right)$. This indicates that another factor is responsible for the increase in $T_{\mathrm{g}}$.

Figure $4 \mathrm{a}$ shows the most probable geometry of BTBB determined by computational chemistry. The geometry can be understood as that in which a pyridyl 
group is deleted from the optimized geometry of tris(terpyridine)-substituted benzene with $C 3$ symmetry. ${ }^{30}$ However, the geometry can be easily converted to others, as shown in Fig. 4b, by rotating bi- or ter-pyridine wings around the single bond connecting the wing with the central phenyl ring. Usually, the energy barrier for such a rotation is only a few times higher than the thermal energy of room temperature $(2.51$ $\mathrm{kJ} / \mathrm{mol}$ ). Figure $4 \mathrm{~b}$ shows the energy difference between the total electron energies of the most stable geometry and the other three geometries. The maximum energy difference is about $2 \mathrm{~kJ} / \mathrm{mol}$. This means that the four geometries should coexist in a solid. This mixing probably also results in the material being amorphous, similar to the symmetrical tris bi- or ter-pyridyl benzenes reported before. ${ }^{30}$ However, four geometries contribute to the amorphous nature of $\mathrm{BBTB}$ and $\mathrm{BTBB}$, while two geometries contribute in the case of the tris-substituted analogues. ${ }^{30}$ This difference between the numbers of contributing geometries is probably one of the reasons why BTBB shows a comparable $T_{\mathrm{g}}$ to tris(terpyridine)-substituted benzene, which has a larger MW than BTBB. This is because crystallizations must require that molecules are in the same conformation. In addition, BTBB has two terpyridine wings, which requires a larger free volume for rotation. In addition, the structures in Fig. $4 \mathrm{~b}$ indicates that one terpyridine must rotate for changing the $3 \mathrm{rd}$ and 4 th lowest geometries to the optimized one. We think that this probably accounts for the perfect amorphous nature of BTBB.

Table 1 also shows the densities of BBTB and BTBB measured in their thin-film states. The densities of BBTB and BTBB are both equal to $1.27 \mathrm{~g} / \mathrm{cm}^{3}$, which is larger than those of tris(bipyridine)- and tris(terpyridine)-substituted benzene $\left(1.21 \mathrm{~g} / \mathrm{cm}^{3}\right)$, which were also measured in this study. These high densities may also contribute to the increase in $T_{\mathrm{g}}$ according to the free-volume theory, ${ }^{32}$ the standard theory for glass 
transition. Briefly, this is because a larger density means a smaller free-volume fraction (ratio of free volume to total volume) if solids are composed of similar elements.

Figure 5 shows the UV-visible absorption and fluorescence spectra of BBTB and BTBB in their thin-film states. The figure also shows their phosphorescence spectra at $77 \mathrm{~K}$. Both materials show optical absorption onsets at a wavelength of about $350 \mathrm{~nm}$ and UV fluorescence peaks at $370 \mathrm{~nm}$. Band gaps $\left(E_{\mathrm{g}} \mathrm{s}\right)$ of the materials were estimated from the onsets of the optical absorption spectra and are summarized in Table 1. The values of $E_{\mathrm{g}} \mathrm{s}$ were as high as about $3.6 \mathrm{eV}$, and the values of ionization potentials $\left(I_{\mathrm{p}} \mathrm{s}\right)$, which were determined by photoemission-electron-yield spectroscopy, varied from 6.3 to $6.5 \mathrm{eV}$. The fact that BTBB has a higher $I_{p}$ than BBTB indicates that the additional pyridyl group makes the highest occupied molecular orbital (HOMO) deeper without affecting $E_{\mathrm{g}}$. This tendency is also observed in the former symmetrical tris bi- or terpyridine-substituted benzenes; ${ }^{30}$ changing bipyridine with terpyridine increased $I_{\mathrm{p}}$ with no change in $E_{\mathrm{g}}$. On the other hand, the resulting electron affinities $\left(E_{\mathrm{a}} \mathrm{s}\right)$ of BBTB and $\mathrm{BTBB}$, estimated by subtracting $E_{\mathrm{g}}$ from $I_{\mathrm{p}}$ for each, were $2.78 \mathrm{eV}$ and $2.91 \mathrm{eV}$, respectively. The higher $E_{\mathrm{a}}$ of BTBB suggests that its lowest unoccupied molecular orbital (LUMO) is deeper than that of BBTB. This can lead to better electron injection, resulting in the potential for reducing the operation voltage. The modestly large $I_{\mathrm{p}} \mathrm{s}$ of BBTB and BTBB probably produce an effect on hole-blocking. Figure 5 also shows their phosphorescence spectra. The phosphorescence spectra are also relatively similar. Estimated triplet band gaps $\left(E_{\mathrm{g}}{ }^{\mathrm{T}} \mathrm{s}\right)$ for BBTB and BTBB are shown in Table 1, where we assumed that each shoulder marked with the arrows in the figure corresponded to each $E_{\mathrm{g}}^{\mathrm{T}}$. 


\section{OLED Performance}

Figure 6a shows $J-V$ curves of the fluorescent devices (test OLED-1 shown in Fig. 2) with BBTB and BTBB. The devices with the new materials showed a lower operation voltage in comparison with the reference device using Alq as the ETM. The voltage reductions reached $1.5 \mathrm{~V}-2.0 \mathrm{~V}$. In particular, the device with BTBB exhibited a very low operation voltage; a current density of $3.5 \mathrm{~mA} / \mathrm{cm}^{2}$ corresponded to a voltage of $3.0 \mathrm{~V}$. In addition, current densities of the device with the new ETMs rapidly increased with a small boost of applied voltage, and the BTBB device reached $J=100$ $\mathrm{mA} / \mathrm{cm}^{2}$ at a mere $4.5 \mathrm{~V}$. These low operation voltages and the rapid increase in current densities with small over-potentials strongly imply that BBTB and BTBB probably possess both good electron-injection properties from the cathode and fast electron mobilities (electron mobility will be discussed later.) In addition, BTBB had a higher $E_{\mathrm{a}}$ than BBTB, which probably caused it to have a lower operation voltage owing to better electron injection from the cathode. Simple estimates of the electron-injection barriers at the interfaces for $\mathrm{BBTB} / \mathrm{Al}$ and $\mathrm{BTBB} / \mathrm{Al}$ are $0.67 \mathrm{eV}$ and $0.80 \mathrm{eV}$, respectively. Here we assumed the work function of the Al cathode combined with the LiF-injection layer was equal to that of pure aluminum $(3.58 \mathrm{eV})$, and even this probably lead to overestimations. These barriers are slightly higher in comparison with those estimated in the same manner for other ETMs reported with good electron-injection properties from the $\mathrm{LiF} / \mathrm{Al}$ cathode. ${ }^{33}$ We think that bipyridine and/or terpyridine can help smooth electron injections owing to their chelating abilities for metal atoms, which can lead to complex formations at the interface, resulting in midgap states.

Figure $6 \mathrm{~b}$ shows luminous efficiency-current density $(\eta-J)$ characteristics of the devices with BBTB and BTBB. The luminous efficiency of the devices with the new 
ETMs were the same as that of the reference device with Alq as the ETM. BBTB and BTBB potentially possess hole-blocking properties because of their large $I_{\mathrm{p}} \mathrm{s}$, but no differences among the devices were detected, because very slow hole mobility in Alq of about $10^{-8} \mathrm{~cm}^{2} / \mathrm{Vs}^{34}$ can practically prevent hole-leaking to the cathode. As shown in the inset of the figure, the electroluminescence (EL) spectrum of the BTBB device indicates that recombination of both charge carriers (electron and hole) should occur in the Alq layer. In addition, in the UV-blue region, there was no EL assigned to other materials, even in the magnified spectrum, which also supports the fact that no chargecarrier leakage occurred.

Figure 7a shows $J-V$ curves of phosphorescent OLEDs (test OLED-2 shown in Fig. 2) with BBTB and BTBB. Here 1,1-bis((di-4-tolylamino)phenyl) cyclohexane (TAPC) was used as the HT material for reducing the operation voltage and preventing triplet energy leakage from the emissive layer to the HT layer because of the material's high hole mobility ${ }^{35}$ and large $E_{\mathrm{g}}^{\mathrm{T}}\left(2.87 \mathrm{eV}^{36}\right)$. The reference device used 1,3,5-tris(1phenyl-1H-benzimidazol-2-yl)benzene (TPBi) as the ETM, which exhibits high electron mobility ${ }^{37}$ and larger $E_{\mathrm{g}}^{\mathrm{T}}\left(2.58 \mathrm{eV}^{38}\right)$ than Irppy $\left(2.42 \mathrm{eV}^{39}\right)$. The phosphorescent OLED with BTBB exhibited a much lower voltage operation than the reference one with TPBi. The reported LUMO of TPBi is $-2.9 \mathrm{eV},{ }^{40}$, and thus, the electron-injection barriers at the interfaces, $\mathrm{BTBB} / \mathrm{LiF} / \mathrm{Al}$ and $\mathrm{TPBi} / \mathrm{LiF} / \mathrm{Al}$, are probably almost the same because $E_{\mathrm{a}}$ of BTBB is $2.91 \mathrm{eV}$. Therefore, the large difference between the operation voltages for both devices suggests that the electron mobility of BTBB should be much higher than that of TPBi. Note that TAPC has an extremely high hole mobility of about $10^{-2} \mathrm{~cm}^{2} / \mathrm{Vs}{ }^{35}$ despite the fact that TAPC is an amorphous organic semiconductor. Thus, the charge balance in the devices, including the BBTB and BTBB devices, is probably 
biased towards being hole-rich, and hence, the recombination zone of holes and electrons would be located in a part of the emissive layer near the interface between the emissive layer and the BBTB, BTBB, or TPBi layer. Given that the luminous efficiency is almost the same for all devices, in this situation this indicates that the new ETMs do not work as energy leakage paths for triplet excitons of Irppy, although the $E_{\mathrm{g}}^{\mathrm{T}}$ of BTBB is neither smaller nor much larger than that of Irppy.

The luminous efficiency of the BTBB device at a current density of 0.11 $\mathrm{mA} / \mathrm{cm}^{2}$ was $47.4 \mathrm{~cd} / \mathrm{A}$, and this efficiency corresponded to an external quantum efficiency (EQE) of $11.9 \%$ under the assumption of perfect Lambertian light distribution. This EQE is common for the conventional Irppy-based phosphorescent OLEDs with a single emissive layer and a hole-blocking layer such as bathocuproine $(\mathrm{BCP})^{30}$, and thus, BTBB should have hole-blocking ability. These results suggest that BTBB can be used as an ETM with hole-blocking ability for phosphorescent OLEDs. In addition, the inset of Fig. 7b shows that the BTBB device exhibited a higher luminous power efficiency than other devices, which is because of its lower voltage operation, while the EQE and luminous efficiency of the BTBB device is the same as those for other devices, as shown in Fig. 7b.

\section{Electron Mobility}

Finally, we would like to briefly discuss electron mobility in the new materials. Figure 8 shows the transient electron photocurrent waveforms, while the inset presents this data using a double logarithmic scale. These waveforms were obtained by time-offlight (TOF) measurement using the BTBB film whose thickness is $5.17 \mu \mathrm{m}$. Although each photocurrent waveform was not clear non-dispersive, we can clearly see that the transit time $\left(t_{\mathrm{T}}\right)$ of the photogenerated-charge carriers-electrons in this case- 
decreased with increasing applied voltages because the photocurrents decayed faster in such a case. In accordance with the conventional method to determine $t_{\mathrm{T}}$ for dispersive transportations, we drew two auxiliary straight lines, as shown in the figure, and determined $t_{\mathrm{T}}$ at the crossing point of the auxiliary lines. Note that the slopes of the two auxiliary lines for each photocurrent are the same and the slopes are larger than -1 for before $t_{\mathrm{T}}$ and smaller than -1 for after $t_{\mathrm{T}}$, respectively, which also support the argument that the observed photocurrents are due to drift motions in the film. If we consider the electron mobility $\mu$ as

$$
\mu=\frac{D d}{t_{T} V} \quad
$$

where $D$ is the distance between the two electrodes $(5.27 \mu \mathrm{m}), d$ is the thickness of the prepared BTBB layer, and $V$ is the applied voltage, we can determine, for example, $\mu$ as $2.8 \times 10^{-4} \mathrm{~cm}^{2} / \mathrm{Vs}$ for an electric field $E=V / D=816 \mathrm{kV} / \mathrm{cm}$. This mobility is 2 orders of magnitude higher than for Alq $\left(2.0 \times 10^{-6} \mathrm{~cm}^{2} / \mathrm{Vs}\right){ }^{41}$ and 3 times higher than for TPBi $\left(9 \times 10^{-5} \mathrm{~cm}^{2} / \mathrm{Vs}\right)^{37}$ at approximately the same electric field. This high mobility probably accounts for the better $J-V$ curves for OLEDs with BTBB in comparison with those of the OLEDs with the corresponding reference. Note that all the transient photocurrent waveforms for both materials are shown in Fig. S1 and S2.

Figure 9 shows the electron mobility as a function of the square root of the applied electric field for several materials, including BBTB and BTBB. Both BBTB and BTBB show mobilities that are about 3 orders of magnitude higher than for Alq. In particular, the electron mobility of BBTB is greater than $10^{-3} \mathrm{~cm}^{2} / \mathrm{Vs}$ at an electric field of greater than or equal to $490 \mathrm{kV} / \mathrm{cm}$. The higher electron mobility of BBTB should contribute to reducing the operation voltage below that of the BTBB devices, but in fact, 
just the opposite occurs. We think that the smooth electron injection at the interface between BTBB and the cathode owing to the relatively large $E_{\mathrm{a}}$ of BTBB (compared with BBTB) can probably account for the reason why the BTBB devices exhibit better $J-V$ characteristics than the BBTB devices in which the electron mobility is two times higher. However, unfortunately, we are unsure at present why BBTB and BTBB exhibited higher mobilities than their symmetric analogues, tris(bipyridyl)benzene (1.4 $\left.\times 10^{-4} \mathrm{~cm}^{2} / \mathrm{Vs}\right)$ and tris(terpyridyl)benzene $\left(3.2 \times 10^{-5} \mathrm{~cm}^{2} / \mathrm{Vs}\right)$ in an electric field of $680 \mathrm{kV} / \mathrm{cm} .{ }^{30}$ The densities of the BBTB and BTBB thin films are higher than those of their symmetric analogues, which we believe may also contribute to their higher electron mobilities, similarly to the case of $T_{\mathrm{g}}$; a higher density leads to a shorter distance between hopping sites. We regret that we do not fully understand why BBTB and $\mathrm{BTBB}$, asymmetric derivatives of trisubstituted benzene with both bi- and terpyridines, exhibit higher densities than their symmetric analogues. This is still a question that goes beyond the frontiers of molecular material science to the best of our knowledge. Note that it was difficult to determine $t_{\mathrm{T}}$, or mobility for BTBB under applied electric fields below $680 \mathrm{kV} / \mathrm{cm}(370 \mathrm{~V})$. On the other hand, $t_{\mathrm{T}}$ for BBTB were successfully determined in smaller electric fields. This difference might imply that there is larger number of electron traps in the BTBB film compared with the BBTB film. Further detailed studies about the point are needed.

\section{Summary}

We have developed new ETMs for OLEDs based on benzene-substituted bipyridine and terpyridine (BBTB and BTBB) groups. From the viewpoint of the tradeoff relationship between mobility and $T_{\mathrm{g}}$ appearing in our former trisubstituted benzenes 
with bipyridine or terpyridine, the new materials could achieve better properties. $T_{\mathrm{g}} \mathrm{s}$ of BBTB and BTBB were $93{ }^{\circ} \mathrm{C}$ and $108^{\circ} \mathrm{C}$, respectively, of which the $T_{\mathrm{g}}$ of BTBB was almost comparable to that of tris(terpyridyl)benzene, although tris(terpyridyl)benzene has a larger molecular weight. This is due to the additional pyridyl group in BTBB. The substitution with both bi- and ter-pyridines leads to conformational variation, and the four conformers have similar energies with the maximum difference less than the thermal energy of room temperature. This conformational variation contributes to the formation of the glassy state. In particular, we found BTBB to be completely amorphous with no melting point. Electron mobilities of BTBB exceeded the order of $10^{-4} \mathrm{~cm}^{2} / \mathrm{Vs}$, while those of BBTB were very high and reached $10^{-3} \mathrm{~cm}^{2} / \mathrm{Vs}$. The reason these mobilities are higher than those of the former trisubstituted derivatives is probably because of the comparably higher densities of BBTB and BTBB thin films. These high mobilities contributed to a low voltage operation; for example, in the case of the Alqbased device with BTBB, current densities of $3.5 \mathrm{~mA} / \mathrm{cm}^{2}$ and $100 \mathrm{~mA} / \mathrm{cm}^{2}$ were reached at voltages of $3.0 \mathrm{~V}$ and $4.5 \mathrm{~V}$, respectively. However, we observed another mobility- $T_{\mathrm{g}}$ trade-off relationship in the new materials. Further investigations are required to promote the material science of amorphous organic semiconductors. $I_{\mathrm{p}} \mathrm{s}$ of BBTB and BTBB are sufficiently deep to confine holes in emissive layers.

\section{Acknowledgements}

The authors would like to thank Riken Keiki and Rigaku for ionization potential and density measurements. This work was supported by Program for Fostering Regional Innovation in Nagano, granted by MEXT, Japan. 


\section{References}

1 J.-h. Choi, K.-H. Kim, S.-J. Choi and H. H. Lee, Nanotech., 2006, 17, 2246.

2 Y. Nakajima, T. Takei, T. Tsuzuki, M. Suzuki, H. Fukagawa, T. Yamamoto and S. Tokito, J. Soc. Inform. Display, 2009, 17, 629-634.

3 K. T. Kamtekar, A. P. Monkman and M. R. Bryce, Adv. Mater., 2010, 22, 572-582.

4 Y. Ohmori, Laser \& Photonics Reviews, 2010, 4, 300-310.

5 C. W. Tang and S. A. VanSlyke, Appl. Phys. Lett., 1987, 51, 913-915.

6 C. Adachi, T. Tsutsui and S. Saito, Appl. Phys. Lett., 1990, 57, 531-533.

7 Y. Shirota, J. Mater. Chem., 2000, 10, 1-25.

8 U. Mitschke and P. Baeuerle, J. Mater. Chem., 2000, 10, 1471 - 1507.

9 S. A. VanSlyke, C. H. Chen and C. W. Tang, Appl. Phys. Lett., 1996, 69, 21602162.

10 M. Thelakkat and H.-W. Schmidt, Adv. Mater., 1998, 10, 219-223.

11 K. Katsuma and Y. Shirota, Adv. Mater., 1998, 10, 223-226.

12 H. Tanaka, S. Tokito, Y. Taga and A. Okada, Chem. Commun., 1996, 2175-2178.

13 M. Ichikawa, K. Hibino, N. Yokoyama, T. Miki, T. Koyama and Y. Taniguchi, Synth. Met., 2006, 156, 1383-1389.

14 Y.-L. Liao, W.-Y. Hung, T.-H. Hou, C.-Y. Lin and K.-T. Wong, Chem. Mater., 2007, 19, 6350-6357.

15 C.-Y. Lin, Y.-M. Chen, H.-F. Chen, F.-C. Fang, Y.-C. Lin, W.-Y. Hung, K.-T. Wong, R. C. Kwong and S. C. Xia, Org. Electron., 2009, 10, 181-188.

16 Z. Jiang, Z. Liu, C. Yang, C. Zhong, J. Qin, G. Yu and Y. Liu, Adv. Funct. Mater., 2009, 19, 3987-3995.

17 C. Adachi, T. Tsutsui and S. Saito, Appl. Phys. Lett., 1989, 55, 1489. 
18 Y. Hamada, C. Adachi, T. Tsutsui and S. Saito, Jpn. J. Appl. Phys., 1992, 31, 18121816.

19 K. Tamao, M. Uchida, T. Izumizawa, K. Furukawa and S. Yamaguchi, J. Am. Chem. Soc., 1996, 118, 11974-11975.

20 M. Uchida, T. Izumizawa, T. Nakano, S. Yamaguchi, K. Tamao and K. Furukawa, Chem. Mater., 2001, 13, 2680-2683.

21 J. Bettenhausen and P. Strohriegl, Adv. Mater., 1996, 8, 507-810.

22 S. B. Heidenhain, Y. Sakamoto, T. Suzuki, A. Miura, H. Fujikawa, T. Mori, S. Tokito and Y. Taga, J. Am. Chem. Soc., 2000, 122, 10240-10241.

23 G. Hughes and M. R. Bryce, J. Mater. Chem., 2005, 15, 94-107.

24 M. Ichikawa, T. Kawaguchi, K. Kobayashi, T. Miki, K. Furukawa, T. Koyama and Y. Taniguchi, J. Mater. Chem., 2006, 16, 221-225.

25 H. Sasabe, E. Gonmori, T. Chiba, Y.-J. Li, D. Tanaka, S.-J. Su, T. Takeda, Y.-J. Pu, K.-i. Nakayama and J. Kido, Chem. Mater., 2008, 20, 5951-5953.

26 Y.-J. Pu, M. Yoshizaki, T. Akiniwa, K.-i. Nakayama and J. Kido, Org. Electron., 2009, 10, 877-882.

27 H. Tsuji, K. Sato, Y. Sato and E. Nakamura, J. Mater. Chem., 2009, 19, 3364-3366.

28 L. Na, W. Pengfei, L. Shiu-Lun, L. Weimin, L. Chun-Sing, L. Shuit-Tong and L. Zengtao, Adv. Mater., 2010, 22, 527-530.

29 S.-J. Su, T. Chiba, T. Takeda and J. Kido, Adv. Mater., 2008, 20, 2125-2130.

30 M. Ichikawa, K. Wakabayashi, S. Hayashi, N. Yokoyama, T. Koyama and Y. Taniguchi, Org. Electron., 2010, 11, 1966-1973.

31 M. Ichikawa, N. Hiramatsu, N. Yokoyama, T. Miki, S. Narita, T. Koyama and Y. Taniguchi, Phys. Status Solidi (RRL), 2007, 1, R37-R39. 
32 L. H. Sperling Introduction to Physical Polymer Science 4th ed.; John Wiley \& Sons: Hoboken, 2006.

33 T. Matsushima, M. Takamori, Y. Miyashita, Y. Honma, T. Tanaka, H. Aihara and H. Murata, Org. Electron., 2010, 11, 16-22.

34 R. G. Kepler, P. M. Beeson, S. J. Jacobs, R. A. Anderson, M. B. Sinclair, V. S. Valencia and P. A. Cahill, Appl. Phys. Lett., 1995, 66, 3618-3620.

35 P. M. Borsenberger, L. Pautmeier, R. Richert and H. Bässler, J. Chem. Phys., 1991, 94, 8276 .

36 K. Goushi, R. Kwong, J. J. Brown, H. Sasabe and C. Adachi, J. Appl. Phys., 2004, 95, 7798-7802.

37 H. Wen-Yi, K. Tung-Huei, L. Yu-Ting, W. Chung-Chih, H. Tsung-Hsi, C. TengChih, W. Ken-Tsung and I. W. Chih, Appl. Phys. Lett., 2006, 88, 064102.

38 H. Fukagawa, K. Watanabe and S. Tokito, Org. Electron., 2009, 10, 798-802.

39 K. Goushi, Y. Kawamura, H. Sasabe and C. Adachi, Jpn. J. Appl. Phys., 2004, 43, L937-L939.

40 X.-M. Yu, H.-S. Kwok, W.-Y. Wong and G.-J. Zhou, Chem. Mater., 2006, 18, 5097-5103.

41 H. Murata, G. G. Malliaras, M. Uchida, Y. Shen and Z. H. Kafafi, Chem. Phys. Lett., 2001, 339, 161-166. 
Table 1. Basic properties of BBTB and BTBB. $T_{\mathrm{m}}, T_{\mathrm{g}}$, and $T_{\mathrm{d}}$ were measured in their solid state. The other properties were measured in their thin-film state.

\begin{tabular}{lccccccc}
\hline Material & $T_{\mathrm{m}}\left({ }^{\circ} \mathrm{C}\right)$ & $T_{\mathrm{g}}\left({ }^{\circ} \mathrm{C}\right)$ & $\begin{array}{c}\text { Density } \\
\left(\mathrm{g} / \mathrm{cm}^{3}\right)\end{array}$ & $I_{\mathrm{p}}(\mathrm{eV})$ & $E_{\mathrm{a}}(\mathrm{eV})$ & $E_{\mathrm{g}}(\mathrm{eV})$ & $E_{\mathrm{g}}{ }^{\mathrm{T}}(\mathrm{eV})$ \\
& & & & & & & \\
\hline BBTB & 226 & 93 & 1.27 & 6.33 & 2.78 & 3.55 & 2.79 \\
BTBB & n.d. & 108 & 1.27 & 6.50 & 2.91 & 3.59 & 2.53 \\
\hline
\end{tabular}

n.d: not detected.
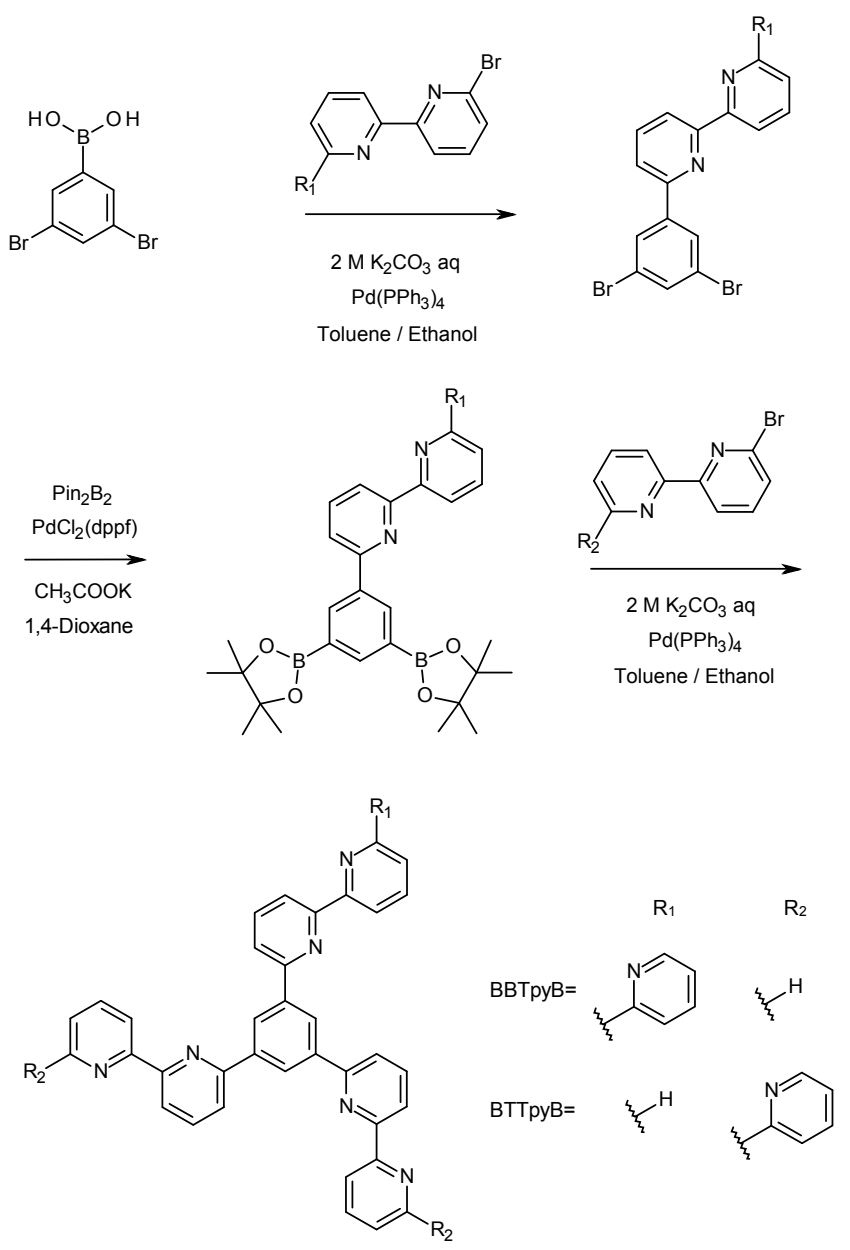

Scheme 1. Synthetic route to BBTB and BTBB. 

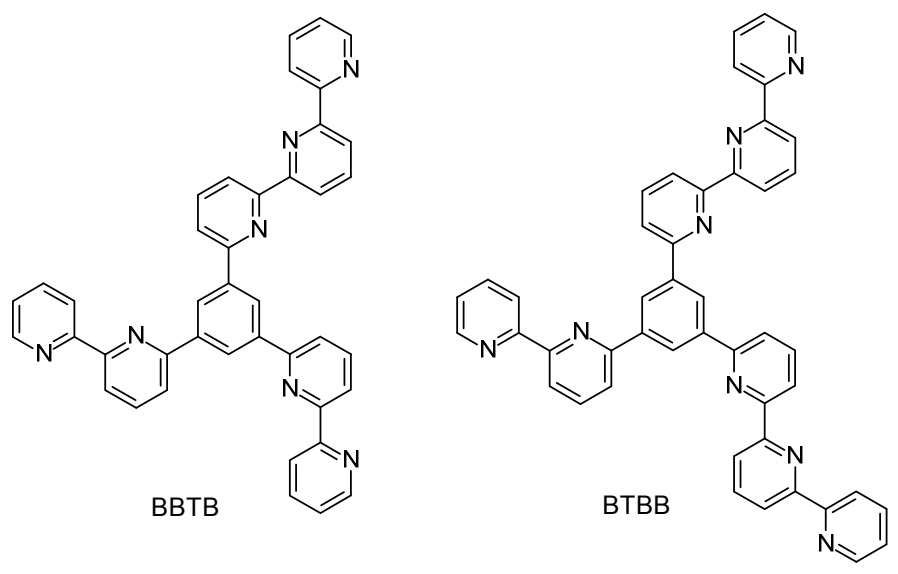

Figure 1. Chemical structures of BBTB and BTBB.

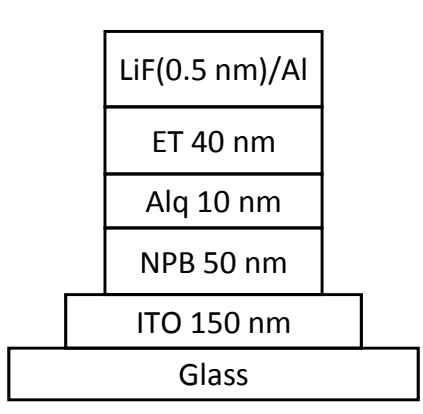

Test OLED-1

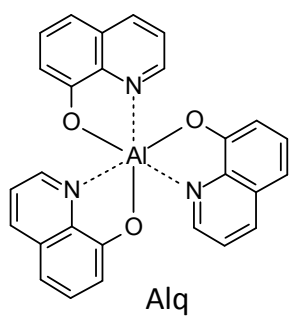

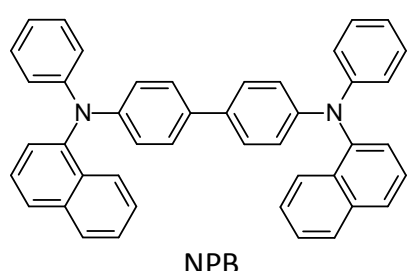

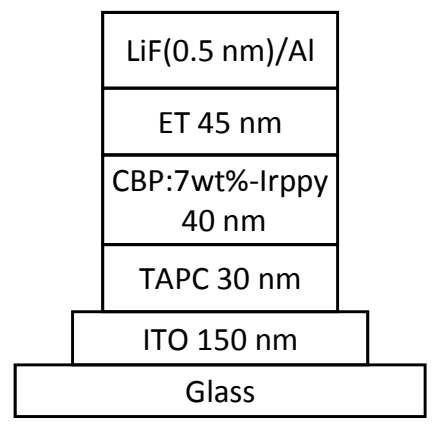

Test OLED-2<smiles>CCCCC(CCCC)(c1ccc(N(c2ccc(C)cc2)c2ccc(C)cc2)cc1)c1ccc(N(c2ccc(C)cc2)c2ccc(C)cc2)cc1</smiles>

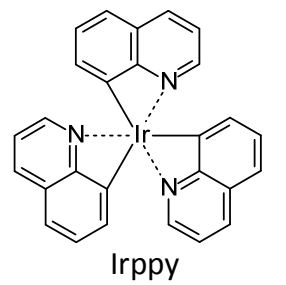

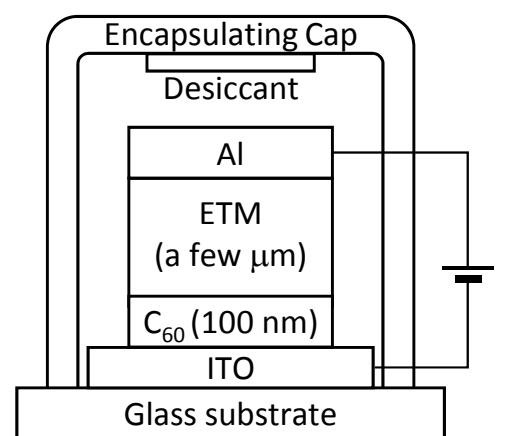

TOF device<smiles>O=C(O)OCc1ccc(-c2ccc(-n3c4ccccc4c4ccccc43)cc2)cc1</smiles>

CBP<smiles>CC(C)C(C)C(C)(C)C(F)(F)c1ccccc1-n1c(-c2cc(-c3nc4ccccc4n3-c3ccccc3)cc(-c3nc4ccccc4n3-c3ccccc3)c2)nc2ccccc21</smiles>

Figure 2. Structures of devices and chemicals used in this study. 


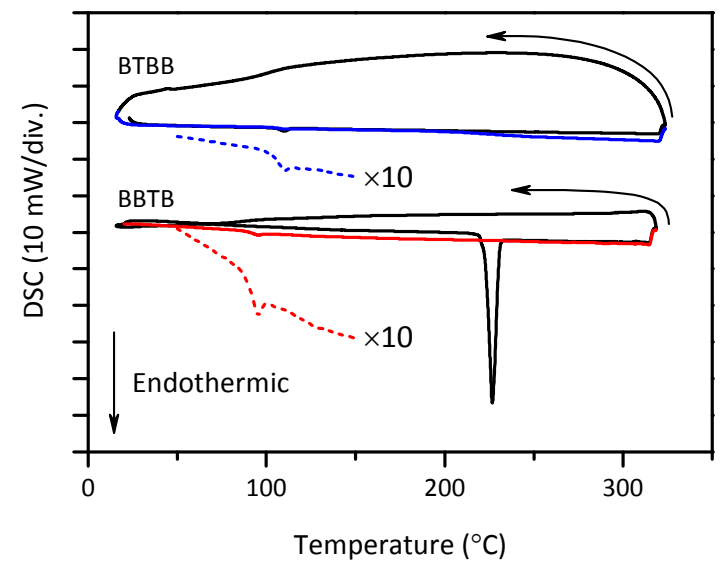

Figure 3. DSC curves of BBTB and BTBB in the first (black line) and second (red for BBTB and blue for BTBB) heating. DSC curves in first cooling are also shown. Dotted lines show vertically 10-times-magnified curves around the temperature range of each $T_{\mathrm{g}}$. Heating rates are 10 and $30{ }^{\circ} \mathrm{C} / \mathrm{min}$ for heating and cooling, respectively. 

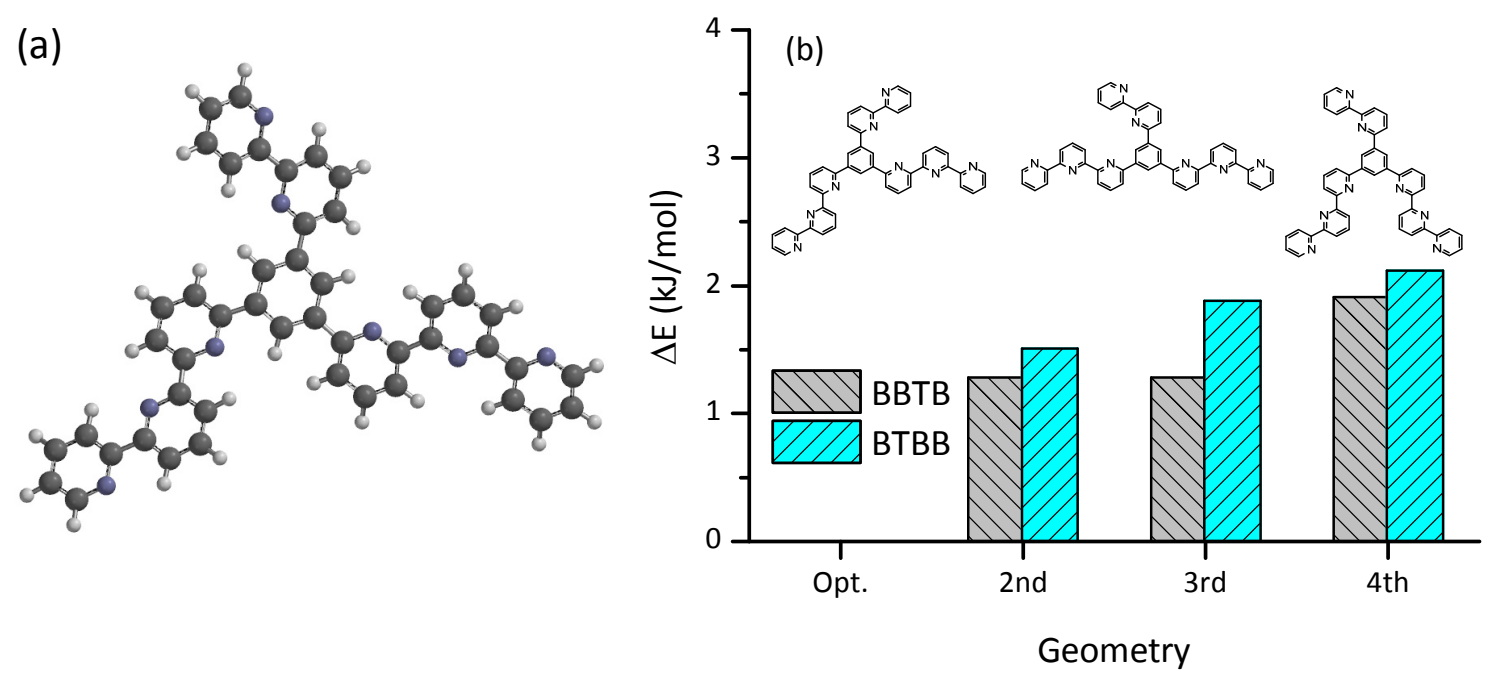

Figure 4. (a) Optimized geometries of BTBB. (b) Difference of total electron energy $(\Delta E)$ of the four most stable geometries for BBTB and BTBB, of which geometries can be mutually converted to each other by rotating the single bonds connecting the coplanar bipyridine or terpyridine wings with the central phenyl ring. The structures in panel (b) represent the geometries of BTBB with the 2nd, 3rd, and 4th lowest energies from left to right. Note that the dihedral angles between the central benzene and the other bipyridine and terpyridine were not zero but almost the same $\left(23-25^{\circ}\right)$. 


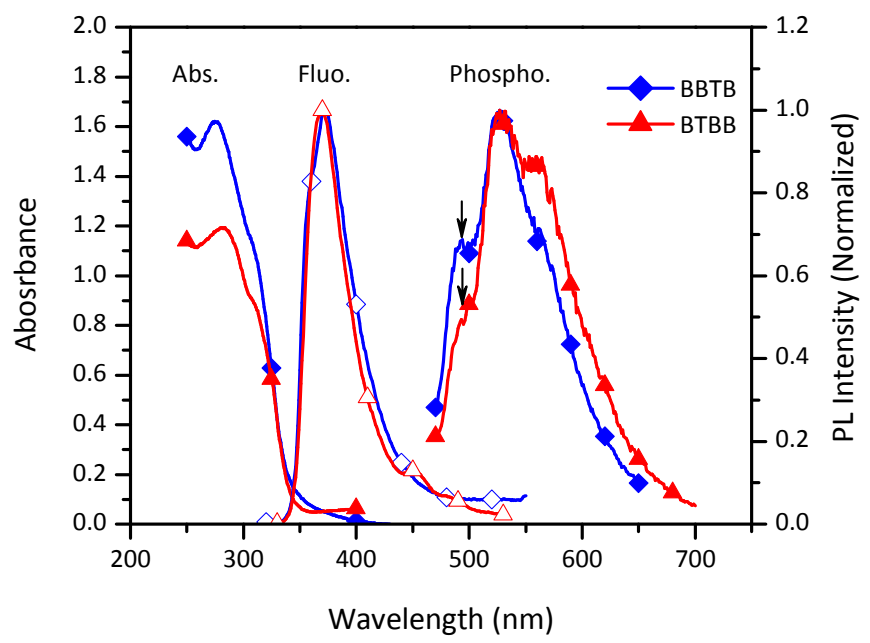

Figure 5. UV-visible absorption, photoluminescence (PL), and phosphorescence (at 77 K) spectra of BBTB and BTBB in their thin-film states. The two arrows are the indications of the peaks used for determining $E_{\mathrm{g}}^{\mathrm{T}}$.
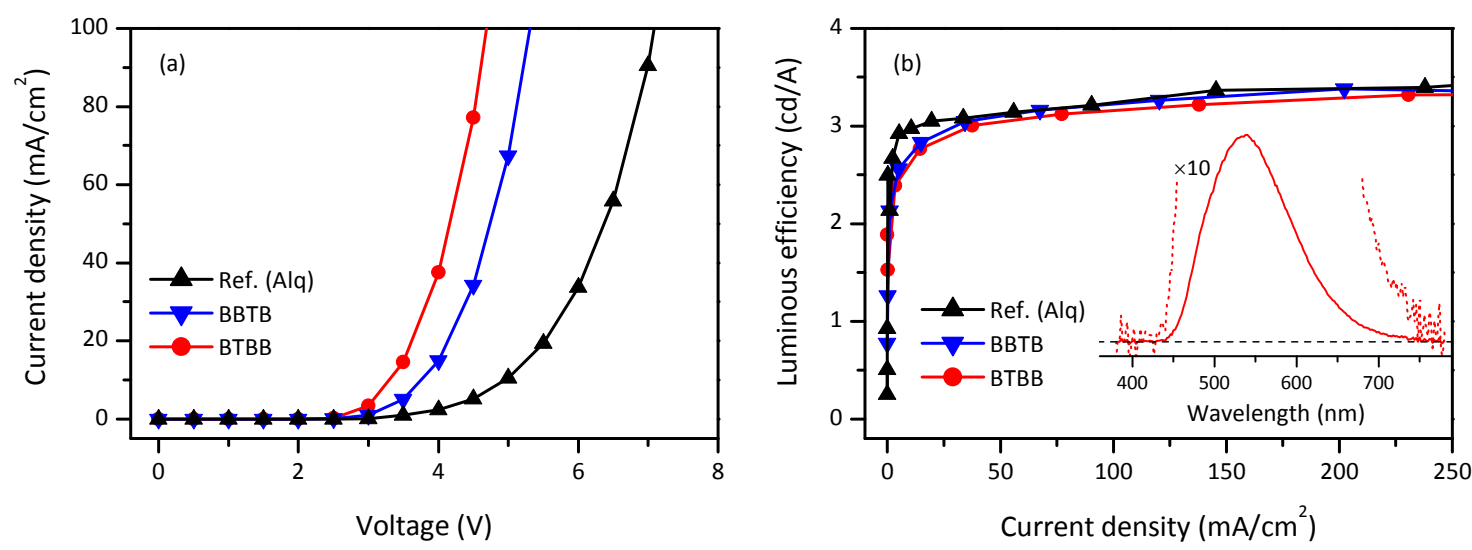

Figure 6. (a) Current density-voltage $(J-V)$ and (b) luminous efficiency-current density $(\eta-J)$ curves of devices with BBTB and BTBB. The inset in panel (b) shows the EL spectrum of the BTBB device at a current density of $80 \mathrm{~mA} / \mathrm{cm}^{2}$. Dashed curve in the inset presents the spectrum magnified by 10 times. 

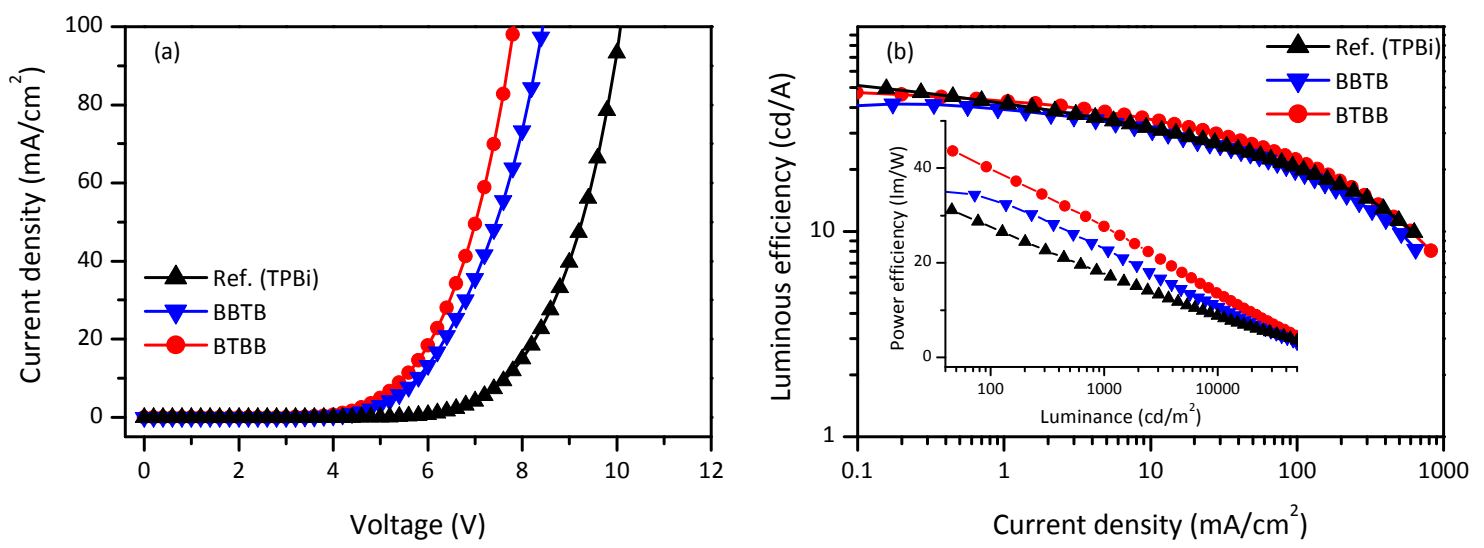

Figure 7. (a) Current density-voltage $(J-V)$, (b) luminous efficiency-current density $(\eta-J)$ and (inset) luminous power efficiency-voltage curves of phosphorescent OLED with BBTB and BTBB.

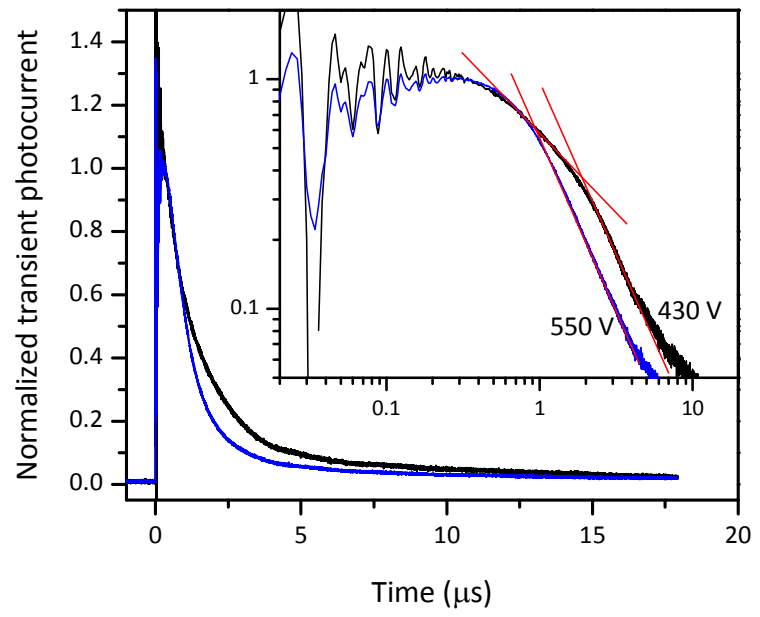

Figure 8. Transient photocurrent curves of TOF measurement of BTBB at applied voltages of 430 and $550 \mathrm{~V}$. Red straight lines in the inset are for guides to determine the transit time. 


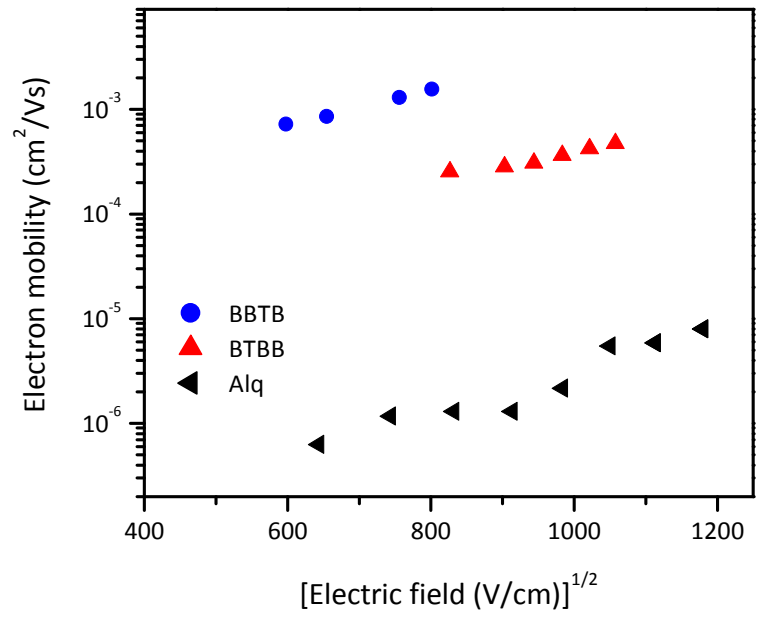

Figure 9. Electron mobilities of BBTB and BTBB as a function of the square root of the electric field. Data for Alq were referred from the literature by Murata (see text). 
Supporting Information

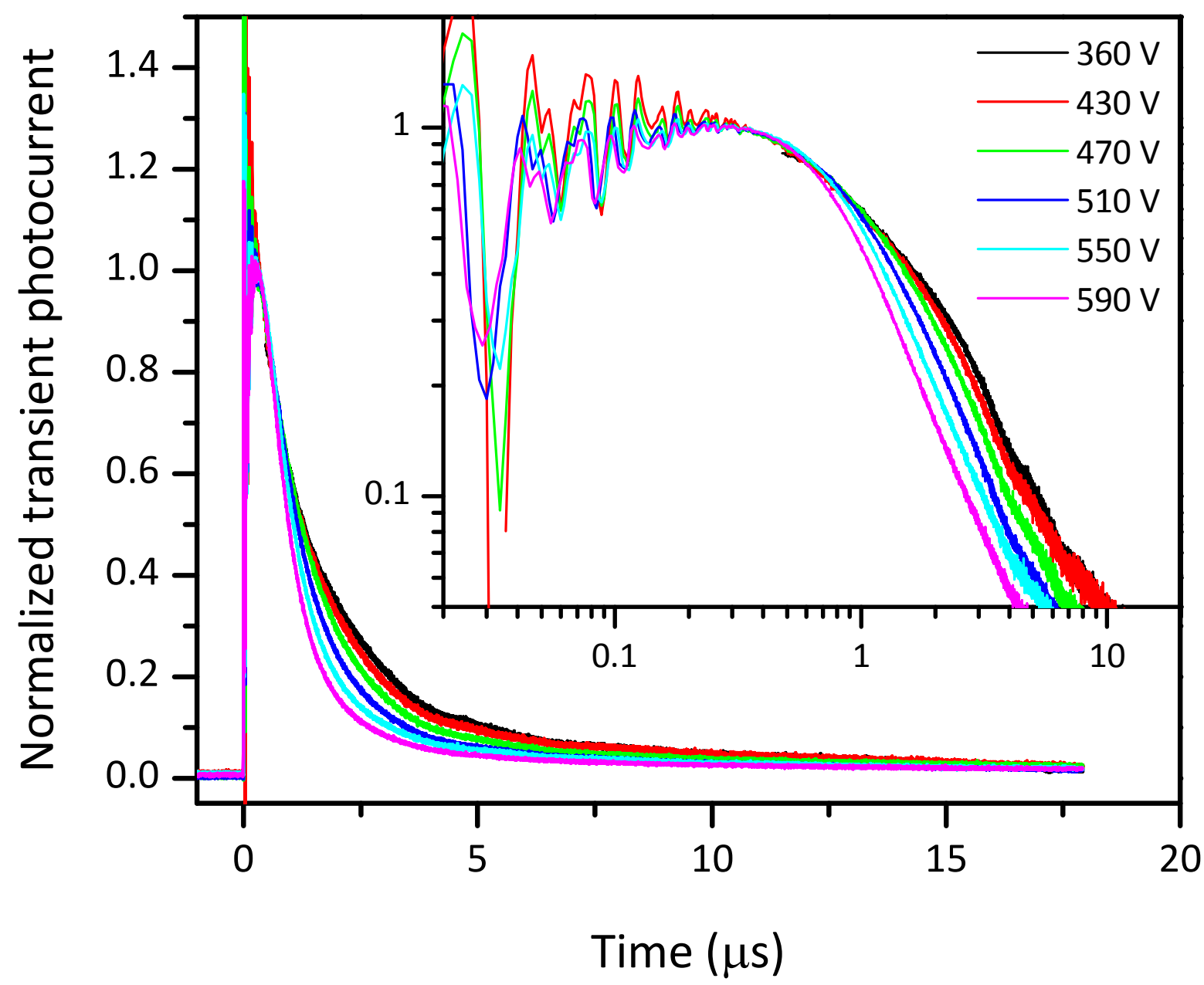

Fig. S1. Normalized transient photocurrent responses for BTBB 


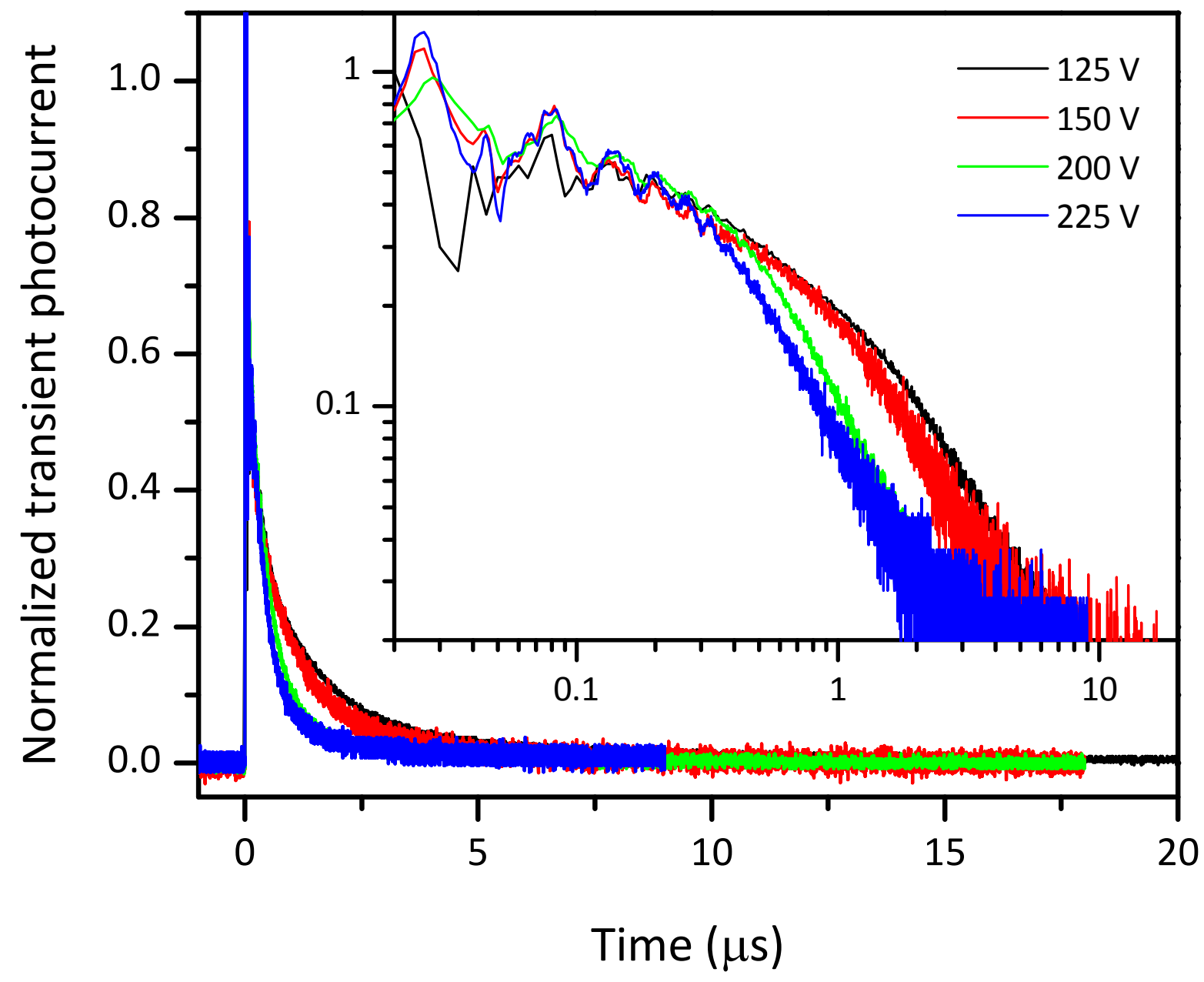

Fig. S2 Normalized transient photocurrent responses for BBTB. The BBTB thickness is 3.40 $\mu \mathrm{m}$. 